\title{
Contributions to the Content Analysis of Gender Roles: An Introduction to a Special Issue
}

\author{
Rena M. Rudy • Lucy Popova • Daniel G. Linz
}

Published online: 31 January 2011

(C) The Author(s) 2011. This article is published with open access at Springerlink.com

\begin{abstract}
This special issue on gender-related content analysis is the second of two parts (see Rudy et al. 2010b). The current special issue is more diverse than was the first in the number of countries that are represented and in the variety of media genres and content types that are included. The primary aim of this paper is to outline some of the contributions of the individual papers in this second special issue. Some of these advancements and innovations include (a) examining underresearched measures, countries, time spans, sexual orientations, and individual media programs; (b) addressing both international and intranational differences in gender-role portrayals; (c) comparing multiple content formats within the same media unit; (d) updating past findings to take into consideration the current media landscape; (e) employing established measures in novel ways and novel contexts; (f) uncovering limitations in established intercultural measures and media-effects theories; (g) suggesting variables that could predict additional differences in gender-role portrayals; (h) adopting virtually identical methods and measures across distinct content categories in order to facilitate comparisons; (i) conducting multiple tests of a given hypothesis; (j) examining, from multiple perspectives, the implications of racial differences in gender portrayals; and (k) examining the implications of underrepresentation of women and the perspectives that women hold. In addition to the original content-analytical research presented in this special issue, two reviews, one methodological and the other analytical, offer recommendations of procedures and perspectives to be implemented in future research.
\end{abstract}

R. M. Rudy $(\bowtie) \cdot$ L. Popova $\cdot$ D. G. Linz

Department of Communication, University of California,

Santa Barbara, CA 93105-4020, USA

e-mail: rena@comm.ucsb.edu
Keywords Content analysis · Gender roles · Feminism · Media effects $\cdot$ Special issue

\section{Introduction}

This special issue on content analysis is the second of two parts, the first having been published in June, 2010 (Rudy et al. 2010a). The introduction to the first special issue (Rudy et al. 2010b) - in addition to discussing historical uses of the content analysis methodology, outlining typical research objectives behind gender-related content analysis, and categorizing typical content analyses of gender rolesprofiled some of the contributions of the individual papers in the first special issue. The purpose of the current introduction is to provide the same type of overview with respect to the individual papers in this second special issue.

The introduction to the first of the two special issues (Rudy et al. 2010b) categorized previous gender-focused content analyses in terms of medium, genre, time span, gender, and nationality, and examined the content analyses in that issue based on those terms. The content analyses in the first group of papers were found to be typical in that most investigated both males and females, and in that many focused on television as a medium, entertainment as a genre, and the United States as a cultural milieu. They were atypical in that film was heavily represented as a medium, and in that multiple studies examined multiple years, encompassing extensive time spans.

The current special issue of Sex Roles on content analysis is, by many criteria, more diverse than was the first. As in the first special issue (Rudy et al. 2010a), media other than television - in this case magazines and newspapers- are represented in half of the original research. Similarly, most articles in both special issues investigated both genders at once. However, in the current issue, one 
content analysis (Schwartz 2010) broached not only gender, but also the issue of gender identity, in examinations of newspaper stories about same-sex marriage. In addition, whereas almost all of the papers in the first special issue limited their formal inquiry to American entertainment, the current articles primarily involve newspaper and advertising content across a wide array of countries, from India to Spain to Canada to Brazil. (All studies involving countries other than the United States have been so identified below; where no country is mentioned, the sole focus was the U.S.) One ambitious research project (Paek et al. 2010) integrated media from seven countries in a single content analysis. Four continents are represented by those countries: Brazil of South America, Germany of Europe, Canada and the United States of North America, and China, South Korea, and Thailand of Asia. One characteristic of the current group of studies that is less diverse than was the first group concerns the time span covered; only three of this issue's studies (Mager and Helgeson 2010; Nam et al. 2010; Schwartz 2010) investigated content that was produced and/or disseminated over the course of one year or longer (though the range of time encompassed by Mager \& Helgeson's analysis, 50 years, is larger than was the largest range in the previous issue). Every article in this special issue, whether a formal content analysis or a review piece, offers a distinct contribution to knowledge about gender-related content.

\section{Content Analysis Articles}

\section{Wallis}

The first two content analyses in this special issue (Turner 2010; Wallis 2010) investigated televised music videos. This content type has been examined in previous studies, but as Wallis remarked, those previous studies generally involved limited samples, broad coding units, passé trends in content, and/or isolated theoretical frameworks. With a goal of "[furthering] knowledge of the media's role in the social construction of gender" (this issue), Wallis sought to overcome many of these limitations by examining performance videos in addition to concept videos, employing relatively small coding units, updating the MTV and MTV2 content under consideration to take into account the current media landscape, and adopting a comprehensive view of Goffman's (1976) gender-display coding framework (in the process repurposing a scheme typically used for coding magazine advertisements as a scheme for coding televised music video content). Wallis's analysis led to the conclusion that in televised music videos, women continue to be portrayed as fragile, subordinate, and sexually suggestive in their behavior, whereas men are generally portrayed as aggressive rather than sexual.
Despite her success in overcoming various limitations of previous research, Wallis (2010) conceded that her own study would be augmented by further content analysis. In particular, Wallis suggested that in future research, attention should be paid to increasing the number of sampled videos, including race of performer as a variable of interest, and considering differences across genres of music.

\section{Turner}

Turner (2010) considered those factors in his analysis of music videos. His first study, of 120 music videos broadcast on five television networks, investigated the relationship between race and gender with respect to sexual display. Given the close association between race and genre in popular music, Turner, like many previous content analysts, used genre of music as a proxy for race of on-screen performers. However, in order to increase the measurement validity of "race," Turner used race of main performer as an additional, more direct, measure. Statistical analyses were conducted with both measures of race. Similarly, two statistical analyses were conducted for each part of the study's second hypothesis; the dependent variable in question, provocative dress, was tested both in terms of frequency of appearance and degree of provocativeness. Doubling the tests of each hypothesis leads to increased confidence in Turner's conclusion that "Black" music videos contain more sexual content than do "White" music videos, as well as in his conclusion that of all race-gender combinations tested, and among both foreground characters and background characters, Black females are shown with the most sexually provocative clothing.

Including multiple tests of a given hypothesis is unusual, as is utilizing state-of-undress measures in race and gender comparisons (see Turner 2010). This paper's contributions to literature on gender and race do not end there; another uncommon feature of Turner's study is an atypical combination of two research goals (see Rudy et al. 2010b): the goal of predicting effects of content on audiences and the goal of predicting effects of content producers on content. The author's concern about effects of content on audiences was manifest in the study's integration of concepts of social cognitive theory (see Bandura 1986) throughout. For instance, one of the theory's propositionsthat frequent exposure to a media stimulus increases behavioral effects - influenced both the choice of television channels to sample (channels were selected primarily based on a pretest assessing the likelihood of the channels' being watched by American college students) and the statistical analysis of the sample (the coding results for a particular video were statistically weighted according to the number of times that video appeared in the overall sample; that is, according to the frequency of exposure that 
audiences would likely have). The author's concern about the influence of content producers on content, on the other hand, was manifest in Turner's discussion of increasing White hegemony in the music industry, and in particular, in his assessment of apparent changes in content that resulted from a Black-to-White change in ownership of BET (the Black Entertainment Television network).

An additional uncommon feature of Turner's (2010) paper is the inclusion of a second, more focused study. Turner added an in-depth analysis of a particular show (BET Un:Cut) on a particular channel (BET) in order to address criticisms of excessive sexuality that have been levied against the show. Recent reports disclose that even a cofounder of that channel, Sheila Johnson, is now "ashamed" of BET because, beginning during her tenure, she "didn't like the way women were being portrayed in [BET music] videos," and because BET "is contributing to an atmosphere of free [unprotected] sex" (Grove 2010, p.1). Turner's second study, of BET Un:Cut, allowed for in-depth analysis that would have been impossible in a simple broad overview of the entire music television landscape. In addition, employing virtually identical methods and measures across the broad first study and specific second study increased the ease (and no doubt, validity) of comparisons across the two samples.

\section{Paek, Nelson, \& Vilela}

Paek et al. (2010) went a step further. Instead of comparing only two samples, these scholars simultaneously scrutinized media in seven countries: Brazil, Canada, China, Germany, South Korea, Thailand, and the United States. The countries represent a wide variety of cultural, historical, geographical, political, economic, and media landscapes, and as Paek and colleagues noted, media content in many of the countries has been underanalyzed. Typically, content analysts aim to uncover and describe gender inequalities by comparing the independent variable of gender with any number of dependent variables. In this study, however, the researchers investigated two characteristics of entire countries (the frequently researched Gender of Nations Masculinity score and the rarely used Gender-Related Development Index score), in addition to a product characteristic (gender of intended product user) in order not only to describe differences across countries of different gender orientations, but also to identify the characteristics that best predict genderrelated inequalities in media content.

The primary advantage of examining many countries at once is the ability to determine whether or not relationships between country and gender inequality that have been found among two or three countries are actually specious. As Paek et al. (2010) remarked, "Examining and comparing multiple countries in crosscultural advertising is beneficial because the number of rival explanations is greatly reduced when the number of cultures involved in evaluating a hypothesis increases" (this issue). Indeed, though research involving pairs of countries has found apparent relationships between a country's "masculinity" and its media portrayals of gender, the current study, sampling a much wider spectrum of national masculinity, found few significant relationships between country-level characteristics and gender portrayals. The strongest predictor of the gender of people portrayed in television ads was not a country-level variable, but was rather a product-level variable; whether the advertised product is typically used by females or males predicted whether the character(s) portrayed (visually or aurally) in an ad would be female or male.

This finding raises interesting questions about the reasons that a product's "gender" (gender of intended product user) tends to match the gender of that product's media spokespeople. Is it because advertisers the world over are sexist; because advertisers understand that some products (e.g., women's lingerie, Viagra) cannot typically be used by people of the opposite sex; and/or because advertisers consciously or unconsciously acknowledge that universal psychological phenomena such as identification, homophily, and parasocial interaction with media characters promote purchase intent (see, e.g., Hoffner and Buchanan 2005; Simpson et al. 2000)? The finding also raises questions about potential solutions to restrictive portrayals of products (e.g., Is using counterstereotypical spokespeople more likely to increase the market size for a product and hence increase profit, or rather repel a product's core market and thereby reduce profit?). This study even raises questions about intercultural research methods (e.g., If a measure of international differences - such as the Gender of Nations Masculinity index - only predicts outcomes among certain select countries, then should that measure be considered valid? Considering that all predictor variables in this study explained only 7 to $15 \%$ of the variance in gender portrayals, should not other predictor variables be considered in crossnational studies?). Among other implications, the findings of Paek et al. suggest that frequently used variables may not adequately explain gender-related differences across national boundaries, even among apparently similar countries.

\section{Das}

Das (2010) agreed (of her subject of study, India) that "findings from other Asian nations may not be entirely generalizable to the Indian context" (this issue), and proffered some variables that may explain additional cross-national variance in gender portrayals. In Indian terms, these variables include religion (Hinduism is the primary religion of the country); Hofstede's (2001) cultural dimensions other than 
masculinity; India's colonial history; Indians' cultural emphasis on family; the low average age of India's population; Indians' relatively young average marriage age; and the fact that television was introduced to India in the 1970s, decades after it was introduced in most of the rest of the world. Das concluded that in India, "there is an indigenously developed sense of modernity with corresponding notions of the 'New Indian Women"' (this issue).

Das (2010) sought to examine this sense of modernity in detail. Complementing Paek et al.'s (2010) broad-brush approach to intercultural issues (i.e., sampling many countries at once and examining few variables in each country), Das examined many variables in a single country, and even addressed intranational differences (by comparing Indian ads geared toward Tamil-speaking audiences with those geared toward English- and Hindi-speaking audiences). This is not to say that Das ignored crossnational comparisons; on the contrary, her stated approach was to follow commonly used research procedures in order to compare her study of India with previously conducted studies of other nations. Comparing primary-source data with data previously published by other researchers allowed for both breadth and depth of analysis. Das concluded that in general, her study's findings mirrored those of Western nations, but that important differences appeared in comparisons between India and other Asian countries.

Nam, Lee, \& Hwang

Like Das (2010), Nam et al. (2010) investigated media in a single Asian country (in the case of Nam et al., South Korea) that is heavily influenced by religious traditions (in the case of Nam et al.'s country of interest, patriarchal Confucianism) but that has recently seen a rapid Westernization. Also like Das, Nam and colleagues were interested not only in intercultural comparisons, but also in intranational differences in gender portrayals. In the study by Nam et al., the latter differences were the primary focus.

The study by Nam et al. (2010) parallels another study in this issue, Turner's (2010), in that race was a variable of chief importance, but the racial perspectives offered by the two studies are in sharp contrast. The country under investigation by Nam et al., the Republic of Korea, can be considered racially opposite Turner's. In the United States, the dominant race is White, while in Korea, White people - and in fact members of all ethnic categories other than Koreans - are in the tiny minority. Nam et al. suggested that the relative homogeneity of Korea's racial makeup offers a potentially illuminating counterpoint to studies conducted in North America, given that racial monoliths such as South Korea might be assumed to demonstrate racially monolithic media portrayals.

Nam et al. (2010) found this assumption to be invalid. Using an adaptation of Goffman's (1976) coding frame- work, the researchers determined that as expected, women were assigned female stereotypes in advertisements published in magazines popular among Korean adolescent girls, and men were portrayed with male stereotypes. However, race portrayals were hardly monolithic; significant differences were found between Asian models and Western models on a number of dimensions, such as skimpiness of attire (with Western women forming the least-clad group). The researchers speculated that skewed portrayals of the White minority in South Korea could lead to harmful stereotypes of White people (perhaps including stereotypes the likes of which have reportedly influenced Middle Eastern terrorists' actions against the U.S., such as the notion that all Whites/Americans are sexually promiscuous and otherwise morally bankrupt - see, e.g., Nisbet et al. 2004; WGBH Educational Foundation 2011). Presumably, an equally probable result for this rapidly westernizing Asian nation, which is ranked highest in the world in per capita plastic surgeries (It's Official 2010), would be that native South Korean adolescents would adopt Western standards of beauty and perhaps seek to westernize their appearance (see, e.g., Cho 2003). Given Nam and colleagues' finding that the prevalence of foreigners has burgeoned to $40 \%$ of Korean advertising models in various indigenous magazines since restrictions on foreign models were lifted in 1989, and given that very few non-Korean, non-White models appeared in their sample, the researchers also expressed concern that young Koreans of other races, or of mixed races, may lack media role models.

Another of Nam et al. (2010) findings led to concern about potential limitations of Goffman's (1976) coding framework. For some variables, males were found to be portrayed in keeping with stereotypes that are "feminine" according to Goffman. Nam et al. noted that these codessuch as open mouths, gaze aversion, and smiling - that detected "female" depictions of men could be variously interpreted as masculine or feminine, depending on the precise nature of the body language. Given that nonverbal communication also differs by culture (e.g., Kirch 1979), interpretation of Goffman's codes likely also depends on culture. Gestures that are seen as feminine in one culture could be seen as masculine in others. An example suggested by Nam et al. is that wearing modest clothing is stereotypically feminine in some Asian cultures, whereas according to Goffman's North American cultural perspective, the opposite - wearing immodest or minimal clothing - is a distinctive practice of female media models. Presumably, interpretation of any given visual signal has also varied over the course of history. It is likely that no set of codes of nonverbal behavior could ever be considered truly universal, but perhaps a refinement of Goffman's framework by international and/or historical scholars is warranted. 


\section{Mager \& Helgeson}

Just as Das's (2010) study of Indian television advertisements (in one country) might be considered an in-depth treatment of part of Paek et al.'s (2010) broad study of television advertisements (in seven countries), so also Nam et al.'s (2010) study of Korean magazine advertisements (over 2 years) might be seen as an in-depth treatment of part of Mager and Helgeson's (2010) broad study of magazine advertisements (over 50 years). Mager and Helgeson noted that magazine advertisements are especially conducive to content analysis because they "provide a 'frozen frame' that allows close visualization and application of a complex code scheme" (this issue), and because magazines have been published, and accessible to vast populations, for over a century. This latter fact allowed the researchers to analyze multiple decades' worth of magazine advertising in a single study; framing their analysis in terms of feminist history and consumerism, the authors examined shifts in gender portrayals over the course of half a century.

Mager and Helgeson (2010) used the same basic Goffman (1976) coding framework that was applied by Nam et al. (2010). The findings of Nam and colleagues led to the suspicion that certain individual variables (e.g., open mouths, eye aversion, and smiling) can reflect gender assignments opposite those suggested by Goffman, but because Mager and Helgeson reported results by Goffman category rather than by individual variable within each category, that claim cannot be tested by Mager and Helgeson's reported findings. The hypotheses that Mager and Helgeson did test produced results that were largely consistent with expectations, at least with respect to overall time-aggregated portrayals of men and women: more often than men, women were portrayed in suggestive poses, in subordinate poses, in withdrawal poses, by only parts of the body, using ritualistic touch, and being instructed (rather than performing an executive role). The only result counter to expectations in the time-aggregated hypotheses was that men did not demonstrate more frequent use of utilitarian touch than did females.

By contrast, only half of hypotheses involving changes over time were supported (Mager and Helgeson 2010). As expected, between 1950 and 2000, the per-advertisement rate of men's appearance in suggestive poses increased, and the incidence of men's performing an executive role decreased. However, a significant increase in female portrayals of utilitarian touch was not found, and the appearance of segments of male bodies (rather than entire male bodies) did not become more frequent over the last half of the $20^{\text {th }}$ century; in fact, men were less likely to be portrayed by only parts of the body around 2000 than they were around 1950 . Perhaps some forms of objectification of male bodies have decreased over the years, or perhaps minimalistic midcentury art styles promoted the use of close-ups of individual faces and hands rather than action involving the entire body.

\section{Matud, Rodriguez, \& Espinosa}

Mager and Helgeson (2010) and Nam et al. (2010) investigated only print advertisements. Matud et al. (2010), by contrast, examined print advertisements as well as articles and photographs - all from the same newspapers - for a more complete picture of gender portrayals in an entire publication. Generally, results were the same across content types (with respect to almost every result that met the assumptions of chi-square testing and that reached statistical significance), and overall, those results demonstrated that males appeared more prominently in newspapers than did females. In each content category - newspaper articles, photographs, and advertisements - males-only content units significantly outnumbered females-only content units. Similarly, according to examinations of content appearing in the top (more prominent) half of the newspaper page, more males than females appeared in newspaper articles and photographs.

The only results that demonstrated greater female prominence detected that prominence in advertising. The sole significant result in analyses of whether or not a content unit occupied an entire newspaper page was found in advertising; more ads featuring women than those featuring men were so large that they filled an entire page. The only result that differed significantly across content types also involved the amount of space a content unit occupied; when measuring prominence by number of columns occupied by a content unit, males were more prominent than were females in articles, and females were more prominent than were males in advertisements. This result does not necessarily indicate that advertisers are more ideologically advanced than are newspaper reporters and editors; without knowledge of the exact nature of these portrayals, this possibility cannot be assessed. Perhaps women were more prominent than were men in advertising because in scanty clothing, women are more easily identifiable as sex symbols; perhaps a "sex sells" ideology rather than a "women are worthy of prominence in society" ideology has taken hold among advertisers in the country under investigation in Matud et al.'s (2010) study: Spain.

Spain is among the many countries whose media content has been underresearched. It is also a country that may be of special interest to gender researchers. For one reason, as noted by Matud et al. (2010), Spain recently emerged from an authoritarian régime and since then has been rapidly assuming democratic Western ideals. This circumstance makes the country a potentially enlightening counterpoint to countries that are still under authoritarian rule, such as China, and to countries from other areas of the world that have also 
been rapidly adopting Western values, such as South Korea. Another reason that gender researchers may find Spain to be an especially interesting subject of study is that during the past decade, Spanish politicians have attempted to legislate gender equality in media. As reported by Matud et al., at least two recent laws include provisions that promote gender equality and prohibit gender discrimination in Spanish media, particularly in advertisements and in content produced by stateowned media. If Spanish legislators are indeed determined to eliminate societal barriers that are based on biological sex, then content analysts interested in extending their research to effect real societal change may find Spain to be an especially hospitable environment.

\section{Schwartz}

In his response to the results of his own content analysis, Schwartz (2010) demonstrated that he is among those researchers who wish to enact change in the journalistic media industry. Schwartz, like Matud et al. (2010), studied underresearched subject matter: the journalistic sources (both heterosexual and homosexual) that were included in newspaper articles involving same-sex marriage. Matud et al. found in their study of all types of Spanish newspaper articles that more males than females were used as sources, and Schwartz found the same to be true of American newspaper articles involving same-sex marriage; males appeared as sources significantly more often than did females among sources overall, among official sources overall, and among homosexual sources in southern and midwestern newspapers. Schwartz's final main finding was that among heterosexual sources, males were significantly more likely to express negativity toward same-sex marriage than were females.

To Schwartz (2010), this combination of findings has potentially profound implications for journalism and audiences' reception to it. The theoretical framework behind Schwartz's study, framing (e.g., Pan and Kosicki 1993), suggests that the sources selected for inclusion in news stories influence the perspectives of the stories and thereby impact people's impression of the issues that are covered. Schwartz speculated that his final finding - namely, that heterosexual males are less in favor of same-sex marriage than are heterosexual females - is materially related to his other findings - namely, that males are more likely than are females to appear as all types of sources in same-sex news stories. If the sources most frequently cited in newspaper sources as a group lean toward the opposing side of an issue, then support for the issue is presumably less likely to grow than it would be if the opinions of supportive groups were more frequently reported. The particular perspectives that are suppressed through strategic or haphazard source selection could potentially skew debate about the issues at hand.
More broadly, Schwartz (2010) suggested that syntactical, thematic, and rhetorical framing devices used by reporters can reveal which members of society the reporters consider to be the most objective, reasonable, and newsworthy. If, as Schwartz and others have found, males greatly outnumber females as journalistic sources, then the assumption reflected in - and, more importantly, perpetuated by - newspapers and their "maleness frame" could be that men are considerably more objective, reasonable, and newsworthy than are females. In addition, the stifling of particular groups in a society through lack of media representation could lead to what a scholar quoted by Schwartz (Tuchman 1978) dubbed "symbolic annihilation"denying certain groups of people a voice in news media could reinforce their lack of importance and power in the functioning of public and private spheres and could result in their virtual annihilation from productive society. Schwartz recommended that future research include interviews with journalists and other observations from newsrooms in order to more closely examine the factors that determine the newspaper sources that are selected for inclusion in newspaper articles (c.f. Desmond and Danilewicz 2010).

\section{Review Articles}

\section{Neuendorf}

The final two articles in this special issue are reviews (rather than reports of original empirical research), one of methodological issues in the content analysis of gender roles (Neuendorf 2010), and the other of general issues raised by the content analyses included in the two special issues (Collins 2011). In introducing her primer on quantitative content analysis methodology, Neuendorf noted that many content analyses lack the methodological rigor that is required to establish the validity of resultseven those content analyses that have been published in peer-reviewed academic journals, and even those content analyses that have been published recently in Sex Roles. Neuendorf's primer is potentially useful for scholars at many stages of content analysis. For gender researchers who have never before performed a content analysis, the primer might serve to pique interest in the research methodology and to provide an introduction to relevant issues. For those who have conducted content analyses in the distant past, the primer may serve to refresh and update previous knowledge. For those who have recently conducted a content analysis that they wish to publish in Sex Roles, the primer may serve to alert the authors to elements of their study that lack sufficient analysis or reportage. Prior to beginning any gender-related quantitative content analysis, and again before submitting a content analysis manuscript to 
Sex Roles or any other academic journal for publication, researchers would benefit from completing a checklist like that outlined in Table 1 of Neuendorf's primer.

Few studies follow all best practices for their respective research methodologies; in reality, practical constraints and hypothesis-driven interests must be balanced with methodological ideals, and difficult decisions must often be made. Nevertheless, the current two special issues on content analysis (see the articles in this issue as well as those outlined by Rudy et al. 2010b) contain several examples of notable decision-making (see Table 1). One laudable methodological decision involves the selection of coders. An ideal of quantitative content analysis is lack of bias in coding, and when gender-sensitive latent content is involved, common sense suggests that the likelihood of bias decreases when equal numbers of each gender serve as coders. (Similarly, if people's valence toward a politically charged topic is assessed - for instance, whether a comment reflects an attitude in favor of, or against, the legalization of samesex marriage - then presumably, the measure would be considered most valid if coders from each side of the political issue were to demonstrate intercoder agreement.) In the current special issues, multiple researchers (Das 2010; Mager and Helgeson 2010) employed equal numbers of male and female coders, and other researchers (Desmond and Danilewicz 2010) ensured that all coders were blind to the study hypotheses. Other exemplary methodological decisions have already been reviewed with respect to articles in the first of the special issues (see Rudy et al. 2010b), but several of these decisions were also made by authors in the current issue. Table 1 lists these decisions (and/or actions based on these decisions), as well as the authors who made them.

\section{Collins}

The conclusion to the two special issues (Collins 2011) is a response to the other papers in the issues as well as a call for new directions in gender-related content analysis theory and research. To Collins, the findings that recurred most often in the special issues are that women are underrepresented in media, that women are sexualized in media, and that women are stereotyped in media. However, Collins noted that important facets of these findings remain undiscovered, and many questions remain unanswered. Do members of underrepresented groups come to identify with dissimilar, overrepresented others despite the psychological impetus to identify with, and learn from, similar models (and if so, do they identify with dissimilar others despite their dissimilarity, or because they come to see

Table 1 Select methodological decisions made by scholars included in the two special issues on content analysis in Sex Roles: A Journal of Research

Beneficial methodological decision

Extending the basic "describe content" goal of content analysis (see Neuendorf 2010; Rudy et al. 2010b)

Directly or indirectly testing theories and/or measures

Explicitly integrating theory into methodological decisions (such as whether or not to include every instance of repeated content units)

Setting a priori criteria for determining whether or not significant statistical differences are substantial enough to be considered practically meaningful

Reducing coder bias

Assessing and reporting unitizing reliability

Conducting multiple tests of a given hypothesis

Conducting post hoc tests to statistically identify the groups that contributed to significant omnibus tests

Using differences of proportion tests to gauge the significance of the differences between particular media content and its real-world equivalent
Example researchers who made the decision

Gilpatric 2010 (used data to create a profile of the prototypical violent female action character); Neuendorf et al. 2010, and Welsh 2010 (used collected data to predict the outcomes of characters' actions); Paek et al. 2010 (used nation-level variables to predict levels of gender equality in media)

Finger et al. 2010 (tested social role theory); Zhang et al. 2010 (tested cultivation theory); Paek et al. 2010 (tested the validity of the Gender of Nations Masculinity score and the Gender-Related Development Index); Nam et al. 2010 (uncovered potential limitations of Goffman's coding framework)

Downs and Smith 2010; Hether and Murphy 2010; Kahlenberg and Hein 2010; Schwartz 2010; Smith et al. 2010; Turner 2010

Downs and Smith 2010; Smith et al. 2010

Das 2010, and Mager \& Helgeson 2010 (employed equal numbers of male and female coders); Desmond and Danilewicz 2010 (ensured that all coders were blind to the study hypotheses)

Das 2010; Hether and Murphy 2010; Neuendorf et al. 2010; Smith et al. 2010

Turner 2010

Das 2010; Downs and Smith 2010; Gilpatric 2010; Nam et al. 2010; Paek et al. 2010; Schwartz 2010; Smith et al. 2010; Turner 2010

Zhang et al. 2010 
themselves as similar to the others)? What other effects does media underrepresentation have (on self-esteem, on knowledge about group-specific health issues, etc.), and how strong are those effects compared with the influence of competing social forces? Is underrepresentation the most pressing issue for gender-and-media scholars, or is it rather the nature of representation when representation does occur (in the case of women, as sexualized and stereotyped)? In other words, is equal (mis)representation a more important and more easily achievable goal, or is it rather positive (under)representation? In what circumstances are oversexed and/or stereotyped portrayals likely to produce negative effects, and under what conditions might they produce positive effects? Collins suggested that the answers to questions such as these might provide content analysts with greater focus and clarity in their research.

Greater focus and clarity might also be promoted by more specific theory. Collins (2011) noted that current theory does not adequately address effects of underrepresentation. Theories of social learning (e.g., Bandura 1977) and theories of identification / homophily / parasocial interaction (see, e.g., Eyal and Rubin 2003) can be co-opted for use in research on underrepresentation, but mediaeffects theories tend to address the nature of portrayals rather than the prevalence of portrayals. Theories native to scholarship on gender representation in media might better answer the above questions.

In her response to the papers included in the two special issues, Collins (2011) called for such theories, and for research aimed at answering questions similar to the above. She also called for greater integration among content analysis, survey, and experimental research methods. Content can be considered irrelevant if it has no influence, and effects produced in experimental laboratories can be considered irrelevant if the content that produced those effects is unlike the specific content to which people are naturally exposed in their living rooms.

Neither are people relegated to their living rooms for massive media exposure any longer. Collins (2011) noted that new media, and the new platforms on which new media are accessed, are ubiquitous; they are also grossly underresearched, as are new methods of interacting with them. As new media become you-media, in the sense that people - both male and female - are increasingly creating and distributing their own media content, Collins asks, "Does this influence gender portrayals in these media?" (this issue).

\section{Conclusion}

This question, and many others, deserve an answer. As we proposed in the introduction to the first of two special issues on content analysis (Rudy et al. 2010b), gender scholars can only benefit from investigating a wide range of media, genres, time spans, genders, and nationalities. Neuendorf (2010) agreed when she asserted, "Scholars may profit by trying to build upon past research by extending the findings of earlier studies to different media, locations, or content forms, or by studying content changes over time" (this issue). As reported above, the scholars included in the two Sex Roles special issues on content analysis (Rudy et al. 2010a, 2011) have done just that. Media analyzed in these special issues include video games, films, television programs, magazines, and newspapers. Locations of origin of the media content include Brazil, Canada, China, Germany, India, South Korea, Spain, Thailand, and the United Kingdom, in addition to all regions of the United States. Content forms include news, music videos, interactive video games, health storylines, violent entertainment, children's entertainment, child-directed advertising, and adult-directed advertising. In six papers (Finger et al. 2010; Gilpatric 2010; Mager and Helgeson 2010; Neuendorf et al. 2010; Smith et al. 2010; Welsh 2010), scholars compared content across as many as 50 years.

Overall, then, the articles included in the two special issues (Rudy et al. 2010a, 2011) are ambitious, comprehensive, and innovative. Some important contributions made by the papers in the current issue in particular include: (a) examining underresearched measures, countries, time spans, sexual orientations, and individual media programs; (b) updating past findings to take into consideration the current media landscape; (c) addressing both international and intranational differences in genderrole portrayals (e.g., Das 2010; Nam et al. 2010); (d) comparing multiple content types within the same media unit (e.g., texts, images, and advertisements in newspapers, Matud et al. 2010); (e) employing established measures in novel ways or novel contexts (e.g., Turner 2010; Wallis 2010); (f) uncovering limitations in established intercultural measures and media-effects theories (see Collins 2011; Paek et al. 2010); (g) identifying variables that could predict important differences in gender-role portrayals (e.g., Das 2010); (h) adopting virtually identical methods and measures across distinct content categories (that span countries, years, types of television programming, etc.) in order to facilitate comparisons (e.g., Das 2010; Mager and Helgeson 2010; Paek et al. 2010; Turner 2010); (i) conducting multiple tests of a given hypothesis (Turner 2010); (j) examining, from multiple perspectives, the implications of racial differences in gender portrayals (Nam et al. 2010; Turner 2010); and (k) examining the implications of the underrepresentation of women and of the perspectives that women hold (e.g., Collins 2011; Schwartz 2010). In addition, the review articles in this issue (Collins 2011; Neuendorf 2010) provide valuable guidelines for conducting content analysis research and for drawing on 
the contributions of the articles in these special issues in such a manner as to profitably expand our knowledge about gender-related media content.

As many authors in these special issues (Rudy et al. 2010a, 2011) attest, plenty of valuable research into genderrelated content has yet to be conducted, but the current special issue and its predecessor provide an abundance of material on which future researchers may profitably draw. The primary aim of this introduction has been to outline some of the contributions of the individual papers in this second special issue, but the contributions made by the articles in these special issues can only be fully appreciated by reading the papers in their entirety.

Acknowledgements We gratefully acknowledge the important contributions of the many people who made these two special issues possible by submitting, reviewing, and publishing papers. We especially appreciate the invaluable guidance given by Irene Hanson Frieze and Susan Dittrich at the Sex Roles editorial office.

Open Access This article is distributed under the terms of the Creative Commons Attribution Noncommercial License which permits any noncommercial use, distribution, and reproduction in any medium, provided the original author(s) and source are credited.

\section{References}

Bandura, A. (1977). Social learning theory. Englewood Cliffs: Prentice Hall.

Bandura, A. (1986). Social foundations of thought and action: A social cognitive theory. Englewood Cliffs: Prentice Hall.

Cho, S. (2003). Beauty is skin deep. The Korea Herald. Retrieved from LexisNexis Academic database, April 8.

Collins, R. L. (2011). Content analysis of gender roles in media: Where are we now and where should we go? Sex Roles, this issue.

Das, M. (2010). Gender role portrayals in Indian television ads. Sex Roles, this issue.

Desmond, R., \& Danilewicz, A. (2010). Women are on, but not in, the news: Gender roles in local television news. Sex Roles, 62, 822-829.

Downs, E., \& Smith, S. L. (2010). Keeping abreast of hypersexuality: A video game character content analysis. Sex Roles, 62, 721-733.

Eyal, K., \& Rubin, A. M. (2003). Viewer aggression and homophily, identification, and parasocial relationships with television characters. Journal of Broadcasting \& Electronic Media, 47, 77-98.

Finger, J., Unz, D. C., \& Schwab, F. (2010). Crime scene investigation: The chief inspectors' display rules. Sex Roles, 62, 798-809.

Gilpatric, K. (2010). Violent female action characters in contemporary American cinema. Sex Roles, 62, 734-746.

Goffman, E. (1976). Gender advertisements. New York: Harper Colophon Books.

Grove, L. (2010, April 29). Sheila Johnson Slams BET. The Daily Beast. Retrieved from http://www.thedailybeast.com/blogs-andstories/2010-04-29/sheila-johnsons-fight-against-hiv-in-dc/

Hether, H. J., \& Murphy, S. T. (2010). Sex roles in health storylines on prime time television: A content analysis. Sex Roles, 62, 810-821.

Hoffner, C., \& Buchanan, M. (2005). Young adults' wishful identification with television characters: The role of perceived similarity and character attributes. Media Psychology, 7, 325-351.
Hofstede, G. (2001). Culture's consequences: Comparing values, behaviors, institutions and organizations across nations (2nd ed.). Thousand Oaks: Sage.

It's official: South Korea has world's highest rate of cosmetic plastic surgery, but... (Summer, 2010). Retrieved from http://www. asianplasticsurgeryguide.com/news 10-2/081003_south-koreahighest.html.

Kahlenberg, S. G., \& Hein, M. M. (2010). Progression on Nickelodeon? Gender-role stereotypes in toy commercials. Sex Roles, $62,830-847$.

Kirch, M. S. (1979). Non-verbal communication across cultures. The Modern Language Journal, 63, 416-423.

Mager, J., \& Helgeson, J. G. (2010). Fifty years of advertising images: Some changing perspectives on role portrayals along with enduring consistencies. Sex Roles, this issue.

Matud, M. P., Rodriguez, C., \& Espinosa, I. (2010). Gender in Spanish daily newspapers. Sex Roles, this issue.

Nam, K., Lee, G., \& Hwang, J.-S. (2010). Gender stereotypes depicted by Western and Korean advertising models in Korean adolescent girls' magazines. Sex Roles, this issue.

Neuendorf, K. A. (2010). Content analysis - A methodological primer for gender research. Sex Roles, this issue.

Neuendorf, K. A., Gore, T. D., Dalessandro, A., Janstova, P., \& SnyderSuhy, S. (2010). Shaken and stirred: A content analysis of women's portrayals in James Bond films. Sex Roles, 62, 747-761.

Nisbet, E. C., Nisbet, M. C., Scheufele, D. A., \& Shanahan, J. E. (2004). Public diplomacy, television news, and Muslim opinion. Harvard International Journal of Press/Politics, 9(2), 11-37.

Paek, H.-J., Nelson, M. R., \& Vilela, A. M. (2010). Examination of gender-role portrayals in television advertising across seven countries. Sex Roles, this issue.

Pan, Z., \& Kosicki, G. M. (1993). Framing analysis: An approach to news discourse. Political Communication, 10, 55-75.

Rudy, R. M., Popova, L., \& Linz, D. G. (2010a). Content analysis [Special issue]. Sex Roles, 62, 705-847.

Rudy, R. M., Popova, L., \& Linz, D. G. (2010b). The context of current content analysis of gender roles: An introduction to a special issue. Sex Roles, 62, 705-720.

Rudy, R. M., Popova, L., \& Linz, D. G. (2011). Content analysis II [Special issue]. Sex Roles, this issue.

Schwartz, J. (2010). Whose voices are heard? Gender, sexual orientation, and newspaper sources. Sex Roles, this issue.

Simpson, E. M., Snuggs, T., Christiansen, T., \& Simples, K. E. (2000). Race, homophily, and purchase intentions and the black consumer. Psychology \& Marketing, 17, 877-889.

Smith, S. L., Pieper, K. M., Granados, A., \& Choueiti, M. (2010). Assessing gender-related portrayals in top-grossing G-rated films. Sex Roles, 62, 774-786.

Tuchman, G. (1978). Hearth and home: Images of women in the mass media. New York: Oxford University Press.

Turner, J. S. (2010). Sex and the spectacle of music videos: An examination of the portrayal of race and sexuality in music videos. Sex Roles, this issue.

Wallis, C. (2010). Performing gender: A content analysis of gender display in music videos. Sex Roles, this issue.

Welsh, A. (2010). On the perils of living dangerously in the slasher horror film: Gender differences in the association between sexual activity and survival. Sex Roles, 62, 762-773.

WGBH Educational Foundation. (2011). What are some typical misperceptions and stereotypes Westerners hold about Islam and the Middle East, and vice versa?. Retrieved from http://www.pbs. org/wgbh/globalconnections/mideast/questions/types/index.html

Zhang, Y., Dixon, T. L., \& Conrad, K. (2010). Female body image as a function of themes in rap music videos: A content analysis. Sex Roles, 62, 787-797. 